\title{
Effect of Structured Teaching Programme on KnowledgeRegarding Passive Smoking Effects in Children among Smoking Parents
}

\author{
Rohini U K Nair ${ }^{1}$, G Sarojini ${ }^{2}$ \\ I'Research Scholar, School of Behavioral Sciences, M G University, Kottayam, Kerala, India) \\ ${ }^{2}$ (Head of the Department of Child Health Nursing, College of Nursing, KMC, KUHS, India)
}

\begin{abstract}
The study attempts to evaluate the effect of structured teaching programme on knowledge regarding effects of passive smoking in children among smoking parents with the objectives to assess the existing level of knowledge among smoking parents, compare the difference between pre-test and post-test knowledge scores of smoking parents and find out the association between the level of knowledge with selected socio-personnel variables and compare the difference between various areas of knowledge scores. The study was carried out in selected areas of AnjarakandyGramapanchayat, Kannur district, Kerala. The sample comprised of 100 smoking parents selected by simple random sampling technique after identification of smoking parents through survey method. Data was collected by administering survey format and structured knowledge questionnaire with the help of structured teaching programme. The results of this study showed that post-test knowledge scores are higher than the pre-test knowledge scores(Good knowledge level increases from 0\% to 48\%). Parents knowledge regarding passive smoking and it's ill effects have significant association with the age, educational status, job, monthly family income of smoking parents and also associated with number of smoking materials per day and period as a smoker. The study findings support the need for STP to improve the knowledge of smoking parents regarding adverse effects of passive smoking and can improve the well-being of the children. Based on the findings of this study some recommendations suggested.
\end{abstract}

Key words: Passive smoking, smoking parents and structured teaching programme.

\section{Introduction}

The differences in children and adult are based on the anatomical, physiological and psychological differences between the immature and mature adults. Children are often more susceptible to health effects of indoor air pollution and passive smoking effects because their immune systems and developing organs are still immature. ${ }^{1}$ Irritation and inflammation caused by air pollution is more likely to obstruct their narrow airways. Exposure to smoke and toxic air contaminate during infancy or childhood could affect the development of respiratory, nervous, endocrine and immune systems and could increase the possibility of cancer later in life. ${ }^{2}$

Children are at higher risk of damage from passive smoking than adults because of their smaller bodies, higher breathing rates and less developed respiratory and immune system. They are more likely to be exposed to cigarette smoke in the home or car, but exposure also occurs in such places as shopping centers, other people's home and social meeting places. ${ }^{3} \mathrm{WHO}$ experts found that around 165,000 children die every year due to passive smoking effects. Passive smoking in children increases yearly. For example, 1, 54, 200 cases reported in 2010 but it increased to $2,00,176$ in $2011 .{ }^{4}$ The IAP estimates that environmental tobacco smoke is responsible for between 150,000 and 300,000 lower respiratory tract infections in infants and children under eighteen months of age annually, resulting in between 7,500 and 15,000 hospitalizations each year. ${ }^{5}$

\section{Statement of the problem}

"A study to assess the effect of structured teaching programme on knowledge regarding effects of passive smoking in children among smoking parents in AnjarakandyGramaPanchayat".

Objectives

1. Assess the level of knowledge among smoking parents regarding the effects of passive smoking in children.

2. Evaluate the effect of structured teaching programme on knowledge of smoking parents regarding effects of passive smoking in children.

3. Associate the level of knowledge of smoking parents regarding passive smoking effects in children and selected socio personnel variables such as sex, level of education etc.

4. Compare the difference between pre-test knowledge scores of smoking parents with areas (sub-sections) of questionnaire. 


\section{Hypotheses}

H1: The mean post - test knowledge scores of smoking parents will be more than their mean pre-test knowledge score.

$\mathrm{H} 2$ :There will be association between pre-test knowledge scores of smoking parents with selected socio personnel variables.

H3: There will be difference between pre-test knowledge scores of smoking parents with areas of questionnaire

\section{Methods And Materials}

\section{Research Approach}

Phase I: descriptive survey approach

Phase II: evaluatory approach

\section{Research design}

Phase I: non-experimental design

Phase II: Pre-experimental, one group pre-test post- test design

Variables

Independent variables: structured teaching programme on knowledge regarding the passive smoking effects in children Dependent variable: knowledge levels of smoking parents regarding passive smoking effects in children.

\section{Schematic representation of the study}

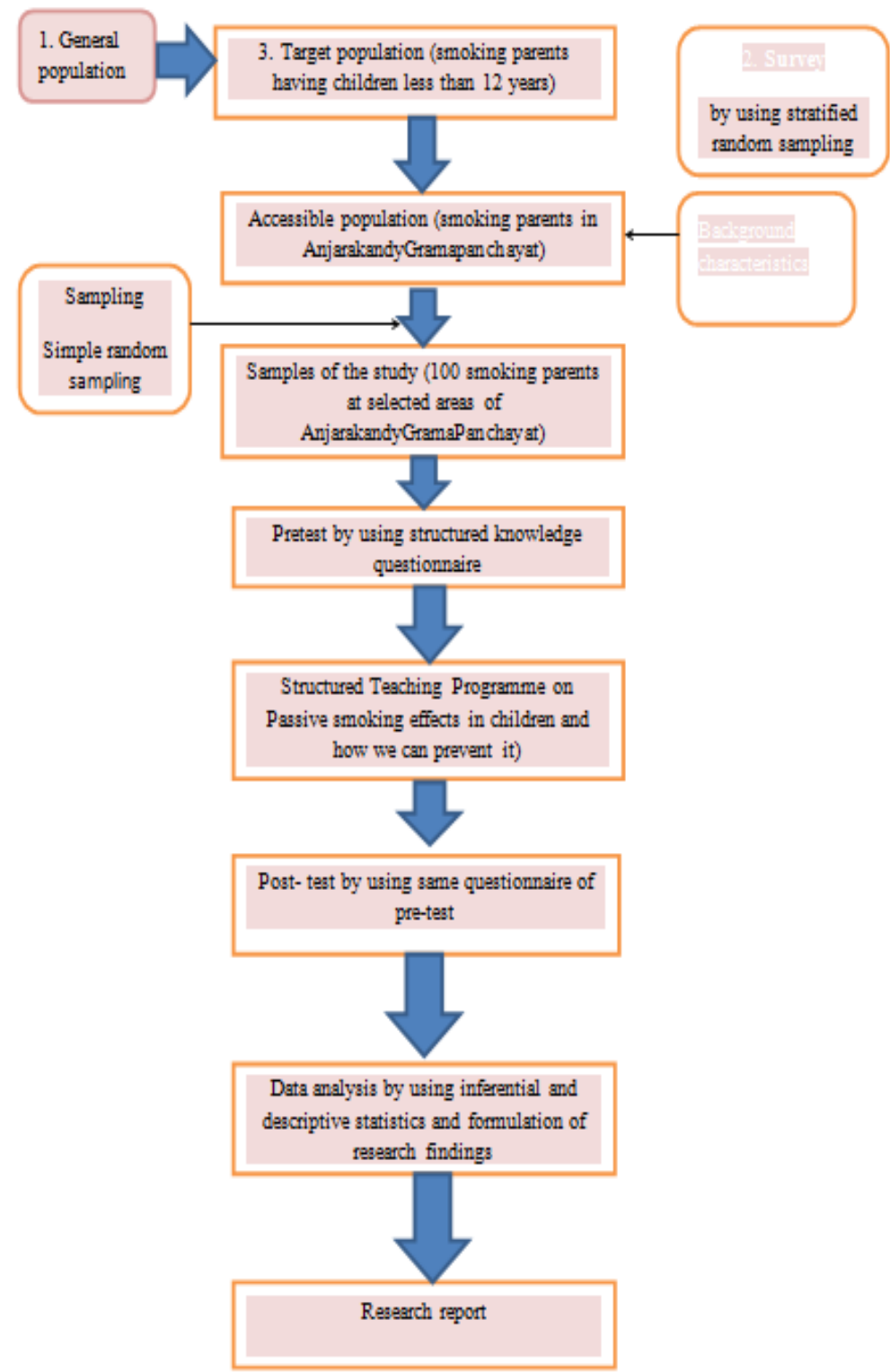




\section{Description of the tool:}

Phase: 1- Survey format (to identify smoking parents and they are having children of age 0-12 yrs.)

Phase: 2- Structured Knowledge questionnaire on passive smoking effects in children. It contains 2 sections.

Section A: Socio - personnel variables (11 questions)

Section B: structured knowledge questionnaire (45 questions)

\section{Description of STP}

Structured Teaching Programme includes three areas Introduction to passive smoking, Passive smoking effects in children and how we can protect the child.

\section{Results}

Section: 1- identify the smoking parents from selected community

- Among 225 houses, 142 houses had smoking parents i.e. $63.11 \%$.

\section{Section: 2- distribution of smoking parents based on socio-personnel variables}

- Mostof the participants (75\%) were in the age group of $>36$ years and $100 \%$ of samples were males and $60 \%$ of them belong to Hindu culture, $(36 \%)$ of samples had only high school qualification, $36 \%$ smoking parents had daily wages and only $16 \%$ had government job, $82 \%$ of study samples coming from BPL families, $55 \%$ of samples had three children, whereas $29 \%$ had two children, $80 \%$ of samples were using cigarettes and remaining $20 \%$ were beedi, $64 \%$ of samples were using 11 to 20 cigarettes per day and $17 \%$ of samples were using cigarettes more than 20 per day and $73 \%$ had history of smoking greater than ten years.

Section:3- find out the existing level of knowledge

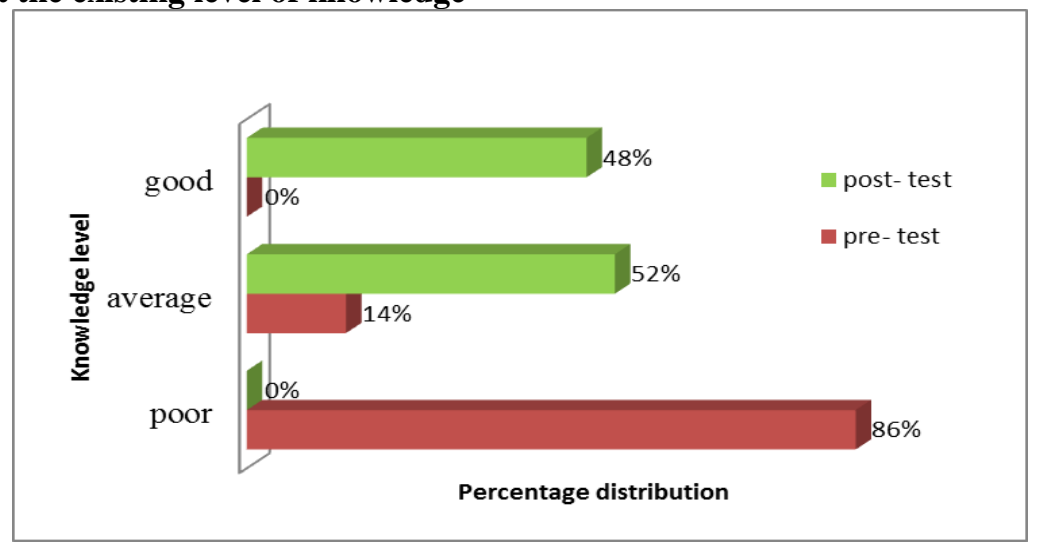

Existing level of knowledge among samples shows $86 \%$ had poor knowledge on passive smoking and its effects in children whereas $14 \%$ had average knowledge score. No one had good knowledge level.

Section:4- evaluate the effectiveness of structured teaching programme

\begin{tabular}{|l|l|l|l|l|l|}
\hline & Mean & N & Standard error & df & Paired t \\
\cline { 1 - 5 } Pre-test & 8.28 & 100 & 174.12280 & 99 & $11.055^{*}$ \\
\cline { 1 - 3 } & 27.53 & & & & \\
\hline
\end{tabular}

The mean post- test knowledge score was higher than the pre -test knowledge level. The calculated paired t' value is 11.055 was greater than the table value (1.66) at 0.01 level of significance. Hence the null hypothesis Ho was rejected and research hypothesis was accepted

Section:5- associate the level of knowledge of smoking parents with selected demographic variables

\begin{tabular}{|c|c|c|c|c|c|c|}
\hline Sl no: & Socio personnel variables & Chi -square & $\begin{array}{l}\text { 'p'(Table } \\
\text { value) }\end{array}$ & $\begin{array}{l}\text { Degree of } \\
\text { freedom }\end{array}$ & $\begin{array}{l}\text { Level of } \\
\text { significance }\end{array}$ & Inference \\
\hline 1 & Age & 12.91 & 12.592 & 6 & $\mathrm{P}<0.05$ & $*$ \\
\hline 2 & Sex & 3.58 & 5.991 & 2 & $\mathrm{p}>0.05$ & NS \\
\hline 3 & Religion & 9.20 & 12.592 & 6 & $\mathrm{p}>0.05$ & NS \\
\hline 4 & Educational status & 19.147 & 16.812 & 6 & $\mathrm{P}<0.01$ & *** \\
\hline 5 & Job & $23 . .904$ & 16.812 & 6 & $\mathrm{P}<0.01$ & $* *$ \\
\hline 6 & Monthly family income & 16.89 & 5.991 & 2 & $\mathrm{P}<0.01$ & $* *$ \\
\hline 7 & Number of children & 2.433 & 12.592 & 6 & $\mathrm{p}>0.05$ & $\mathrm{NS}$ \\
\hline 8 & Type of tobacco used & 12.953 & 12.592 & 6 & $\mathrm{P}<0.05$ & $*$ \\
\hline 9 & Number of smoking material per day & 14.649 & 12.592 & 6 & $\mathrm{P}<0.05$ & $*$ \\
\hline 10 & Period as a smoker & 21.67 & 16.812 & 6 & $\mathrm{P}<0.01$ & *** \\
\hline
\end{tabular}

df $(2)=5.991, \mathrm{P}<0.05 / \mathrm{P}<0.01, \mathrm{df}(6)=16.812, \mathrm{P}<0.05 / \mathrm{P}<0.01, *, * *$ significant and NS - non significant 
Table shows Age(12.91), type of tobacco used (12.53) and number of smoking materials per day (14.649) were significant at 5\% level of significance.Educational status (19.149), job (19.147), monthly family income (16.49) and period as a smoker (21.67) were highly significant at $1 \%$ level of significant. Similarly type of tobacco used (12.53) and number of smoking materials per day (14.649) were also significant at $\mathrm{p}=0.05$. Whereas educational status (19.147), job (19.147), monthly family income (16.89) and period as a smoker (21.67) were highly significant at $1 \%$ level of significant.So the null hypothesis $\mathrm{Ho}$ rejected for this variable and $\mathrm{H} 2$ accepted i.e. there was significant association of selected socio personnel variables with pre-test knowledge score.

Section: 6- compare the difference between pre-test knowledge scores of smoking parents with areas (subsections) of questionnaire.

\begin{tabular}{|l|l|l|c|c|}
\hline Source of variation & Squares of variance & Degree of freedom & Mean square & F-rate \\
\hline Between samples & 3966.1 & 99 & 40.949 & $2.10^{*}$ \\
\hline Within the samples & 3815.57 & 201 & 19.07 & \\
\hline
\end{tabular}

The F score (2.10) was more than the table value (1.25) at $5 \%$ level of significance. Hence the null hypothesis Ho rejected and $\mathrm{H} 3$ accepted. That means each area of questionnaire had their own importance and individual role.

Nursingeducation

\section{Nursing Implication}

- Emphasis more on improvement of public awareness regarding illness and preventive measures

- Equipping the nursing curriculum with dissemination of health information using various methods of educational technology.

- Educating the community people regarding the ill-effects of passive smoking in children and preventive measures to reduce passive smoke.

Nursing practice

- Identify the needs of community children and impart knowledge to smoking parents regarding the effects of passive smoking in children and how we can prevent it

- On anti-tobacco day like celebrations we can conduct in community settings along with awareness class and exhibitions.

- Quality care activities can be initiated

Nursing administration

- Health policy, developing protocols, procedures and standing orders related to preventive measures of passive smoking

- The nurse administrators could organize a screening programme with health education programme on passive smoking effects in children.

- Arrange a health exhibition, workshops, conferences, and discussion in collaboration with other allied health science to make more aware about the different aspects of passive smoking effect in children.

Nursing research

- Large scale studies can be conducted by following various methods of researches.

- Study findings will help to expand the scientific body of knowledge upon which further researches can be conducted

- $\quad$ Findings of the study show that the area needs further exploration

\section{Conclusion}

The pre-test knowledge scores of subjects regarding home based self -care was poor for majority of cases. The introduction of structured teaching programme helped them to learn about passive smoking effects in children and how we can prevent it, which is evident in the post test score.Some socio-personnel factors had strong association with knowledge level of samples.(e.g. age, educational status, job, monthly family income, period as a smoker).

\section{References}

[1] SSPakhale and G BMaru, Distribution of major and minor alkaloids in tobacco, mainstream and sidestream smoke of popular Indian smoking products,J of Food and Chemical Toxicology,36, 1998, 1131-1138.

[2] P K Srivastava, GGPandit, S Sharma and Mohan Rao AM, Volatile organic compounds in indoor environments in Mumbai, India, The Science of Total Environment 2000, 161-168.

[3] D Behera, PSoodand SSinghi,Passive smoking, domestic fuels and lung function in north Indian children. Indian Journal of Chest Diseases and Allied Sciences, 40, 1998, 89-98

[4] R Doll, B Hill, [Jun 2004]. The mortality of doctors in relation to their smoking habits: a plreliminary report. 1954 BMJ [Clinical research ed.\} 328 [7455]. 1529- 1533; discussion 1533. doi: 10.1136/bmj. 328.7455.1529. ISSN 0959-8138. PMC437141. PMIC15217868. http://www.pubmecentral.nih.gov/articlerender.fcgi >tool=pmcentrez\&artid=437141.edit

[5] D Gupta, A NAggarwal, R Kumar and S K Jindal, Prevalence of Bronchial asthma and association with environmental tobacco smoke exposure in adolescent school children in Chandigarh, North India. Journal of Asthma, 38, 2001, 501-507. 